\title{
Family history in primary care: understanding GPs' resistance to clinical genetics - qualitative study
}

\author{
Jonathan Mathers, Sheila Greenfield, Alison Metcalfe, \\ Trevor Cole, Sarah Flanagan and Sue Wilson
}

\section{ABSTRACT}

Background

National and local evaluations of clinical genetics service pilots have experienced difficulty in engaging with GPs.

Aim

To understand GPs' reluctance to engage with clinical genetics service developments, via an examination of the role of family history in general practice.

Design of study

Qualitative study using semi-structured one-to-one interviews.

Setting

The West Midlands, UK.

\section{Method}

Interviews with $21 \mathrm{GPs}$ working in 15 practices, based on a stratified random sample from the Midlands Research Practices Consortium database. Thematic analysis proceeded alongside data generation. Framework grids were constructed for comparative analytical questioning. Interpretation was framed by two explanatory models: a knowledge deficit model, and practice and professional identity model.

\section{Results}

There is a clear distinction between the routine use and function of family history in GPs' clinical decision making, and contrasting conceptualisations of genetics and 'genetic conditions'. Although genetics is clearly a part of current GP practice, with acknowledgement of genetic components to multifactorial disease, this is distinguished from 'genetic conditions' which are seen as rare, complex single-gene disorders. Importantly, family history takes its place within a broader notion of the 'family doctor' that interviewees identified as a key aspect of their role. In contrast, clinical genetics was not identified as a core component of generalist practice.

\section{Conclusion}

The likely effectiveness of educational policy interventions aimed at GPs that focus solely on knowledge deficit models, is questionable. There is a need to acknowledge how appropriate practice is constructed by GPs, within the context of accepted generalist roles and related identities.

Keywords

genetics; physicians, family; qualitative research.

\section{INTRODUCTION}

Knowledge of the genetic contribution to a range of common conditions is increasing. The 2003 White Paper Our inheritance, Our future acknowledged that genetics will feature across the health service, impacting on most healthcare professionals. ${ }^{1}$ It suggested a need to 'mainstream' clinical applications, focusing on single gene disorders and common multifactorial conditions with a genetic component. In response, expanded clinical genetics services have been funded, and pilots of new service models have been undertaken. ${ }^{2}$ In particular, primary care and general practice have been identified as appropriate sites for these developments in clinical genetics service models:

'GPs, practice nurses, and other primary care practitioners will all be able to help their patients benefit from the new genetic knowledge and its applications."

However, GPs seem to have been reluctant to embrace this proposal for an extended role. ${ }^{3,4} \mathrm{~A}$ recent national evaluation of NHS genetics service

J Mathers, research fellow, Public Health, Epidemiology and Biostatistics; S Greenfield, senior lecturer; A Metcalfe, senior research fellow; $S$ Flanagan, research associate; $S$ Wilson, professor, Primary Care Clinical Sciences, University of Birmingham. T Cole, consultant clinical and cancer geneticist, West Midlands Regional Clinical Genetics Service, Birmingham Women's NHS Foundation Trust.

\section{Address for correspondence}

Jonathan Mathers, Public Health, Epidemiology and Biostatistics, University of Birmingham, Edgbaston, Birmingham, West Midlands, B15 2TT.

E-mail: j.m.mathers@bham.ac.uk

Submitted: 26 June 2009; Editor's response: 27 August 2009; final acceptance: 4 February 2010.

@British Journal of General Practice.

This is the full-length article of an abridged version published in print. Cite this article as: Br J Gen Pract 2010; DOI: 10.3399/bjgp10X501868. 
investments highlighted the difficulties that pilot sites had encountered while attempting to engage local stakeholders in primary care:

\section{'A significant and ongoing problem for the pilots centred around the engagement of external stakeholders in primary care, especially GPs. For many of the pilots, primary care was to be the principal or sole source of referrals to the service, but securing the compliance of GPs was a challenge that took much time and effort, if it was surmountable at all. ${ }^{3}$}

Examination of the existing literature attempting to provide explanatory models for GPs' genetics experience, knowledge, and attitudes broadly reveals two schools of thought around barriers to an enhanced role in clinical genetics. First, it has been proposed that GP reactions can be conceptualised within a knowledge deficit model. ${ }^{5-9}$ Here GPs' reluctance to engage with an enhanced role in genetics is tied to a lack of knowledge, skills, and confidence in undertaking required tasks, such as risk assessment, and counselling. Consequently, proposed interventions to improve engagement are primarily educational in nature, while recognising that service developments must accommodate GPs' ways of working, acknowledging, for example, time and workload constraints. ${ }^{10,11}$

The second position challenges the validity of this interpretation. It argues that GPs' attitudes to clinical genetics need to be understood within the context of GPs' day-to-day practice and accepted generalist roles. ${ }^{12,13}$ Theoretical perspectives from the sociology of the professions suggest that knowledge acquisition (for example, genetic knowledge) is actually driven by the requirements of practice, and in turn that such practice is strongly influenced by accepted roles and professional identities. ${ }^{13}$ In other words, GPs acquire knowledge as and when they see it benefiting their practice, and within a framework that is structured by their identity as 'generalists'. Accordingly, education per se is unlikely to change attitudes or practice substantially if those changes do not fit with established and accepted roles, and related professional identities.

Critically, these interpretations of why GPs are reluctant to engage with clinical genetics lead to different interventions to support policy initiatives. To date, knowledge deficit models have been dominant and this is reflected, for example, via the establishment of the National Genetics Education and Development Centre, and the formulation of genetic curriculum statements in undergraduate and postgraduate training. ${ }^{14-16}$ However, observed difficulties in integrating genetics into mainstream

\section{How this fits in}

Most GPs do not seem to have welcomed an enhanced role in clinical genetics. Previous work examining GPs' genetics experience, knowledge, and attitudes has either suggested that this is explained by clinical genetics knowledge deficit, or that GPs' professional identities and experience influence practice and therefore attitudes to genetics. This research shows that although genetics is already implicit within GP practice, the use and role of family history in primary care is distinct from that within clinical genetics. It suggests that knowledge deficit models are inadequate to explain GPs' reactions, and that educational interventions alone are unlikely to have the impact that genetic policy initiatives desire.

health care to date, suggest that knowledge deficit models do not fully explain GP behaviour.

One way to 'test' the interpretations outlined above is to examine how 'genetics' is used by GPs in their everyday practice. Family history is the key diagnostic tool for clinical genetics, and the means by which GPs might identify patients suitable for referral to clinical genetics services. ${ }^{17,18}$ However, there has been little research examining the routine 'practice' of family history in primary care, to understand whether and how GPs already 'do' genetics within this, and, in turn, if this differs substantially in nature from roles they may need to undertake to engage with new clinical genetics service models.

This study aimed to investigate how GPs say they are currently using family history, how genetics is conceptualised within this, and how GPs see clinical genetics impacting on their practice. As a result it is hoped the study will provide a better understanding of whether GPs' reticence to engage fully with clinical genetic developments is a consequence of knowledge deficit, or a rational reaction within the context of generalist practice, roles, and related identities.

\section{METHOD}

Qualitative in-depth interviews were conducted with GPs in the West Midlands, UK.

\section{Sampling}

The Midlands Research Practices Consortium (MidRec) database was used as a sampling framework. ${ }^{19}$ MidReC consists of a network of over 600 practices, covering a representative population of over 4 million residents of the West Midlands. To include GPs working in a variety of settings the database was stratified by practice list size, ethnicity, and deprivation, prior to randomly selecting practices to be approached. Practice managers in 100 practices were contacted, the nature of the study explained, and written information was 
forwarded to be distributed among practice GPs. Those practices that had not responded within 2 weeks were telephoned to ascertain if GPs were willing to participate. Practices were offered $£ 75$ per hour per participant (the standard per hour locum payment for MidReC practices). Recruitment was phased to accommodate the analytical approach and ceased once saturation of major themes and conceptual categories was apparent during interview conduct and subsequent analysis. Four GPs with connections to Primary Care Clinical Sciences at the University of Birmingham took part in initial interviews that were intended to refine the interview process and schedule. These have been included in the core dataset, as no major changes to the interview content were required following this pilot phase.

\section{The interviews}

Interviews were conducted at a time and venue chosen by participants (for example, surgery, home, and university). Prior to commencement, the interviewer re-iterated the nature of the study and gave a broad outline of interview content, answered questions, and took consent to participate. The interviewer, an experienced qualitative researcher, is a non-clinical research fellow, without detailed knowledge of clinical genetics. This, and their role as the 'qualitative researcher' in the project, was discussed with participants prior to the interview in an attempt to pre-empt any inhibitive social dynamics. The in-depth interviews were semistructured around a core set of topics (Box 1). Interviews were digitally recorded and their duration ranged from 40 to 84 minutes. The interviewer took notes during and immediately following the interviews.

\section{Data analysis}

Thematic analysis proceeded alongside data generation, to both inform interview conduct and judge saturation. This ensured completeness of the dataset by providing an awareness of emerging analytical questions to be addressed in subsequent interviews. All members of the research team read transcripts from a selection of the initial interviews and met during and immediately following data collection to discuss emerging findings and analytical questions. This provided multiple perspectives on the dataset. The interviewer conducted the main analysis alongside this process. Digital recordings were transcribed verbatim, and analysis was undertaken with reference to the recordings, transcripts, and interview notes. Withincase coding and categorisation was undertaken prior to building a cross-case thematic framework capable of comparative analytical questioning. A thematic grid akin to that described within framework analysis was built up to enable cross-case comparisons based on sample characteristics, while retaining the ability to check within-case contextual validity. ${ }^{20}$

\section{RESULTS}

Twenty-one GPs took part in interviews (Table 1), from 15 practices. A pictorial representation of the thematic structure and relationships within the data is provided in Figure 1. Key to understanding these data, and providing interpretations of them, is the distinction between the routine use and function of family history in GPs' clinical decision making, and the contrasting conceptualisations of genetics ('the G word', interviewee 2) and 'genetic conditions'.

This distinction will be characterised, prior to outlining a broader conceptualisation of the role of family history in generalist practice, and suggestions will be made about how knowledge relevant to family history/genetics in primary care is accrued. Some of the concerns expressed by GPs regarding an enhanced role in clinical genetics will also be outlined, and a description will be given of how clinical cancer genetics is discussed by GPs.

\section{Family history in clinical decision making: use,} utility, and conceptualisation

Interviewees described using family history to inform their clinical judgement in deciding on management

\section{Box 1. Topics covered in the interview.}

- GP - role within practice, working history

- Practice - description (for example, size, number of partners, patient population)

- Family history - use within clinical practice, practice policies, range of relevant patients, clinical scenarios, contribution to decision making and patient management

- Conceptualisations of genetics within the use of family history

- Impact of genetics and advances in genetic knowledge on clinical practice/general practice/patients

- Perspectives on an enhanced role for clinical genetics within primary care

- Relationship between genetic advances and generalist roles and identities 
Table 1. Interviewee and practice characteristics.

\begin{tabular}{|c|c|c|c|c|c|}
\hline GP & Male/female & $\begin{array}{c}\text { Number of } \\
\text { years as GP }\end{array}$ & $\begin{array}{c}\text { List size } \\
\text { (large/small) }^{\mathrm{a}}\end{array}$ & $\begin{array}{c}\text { Practice } \\
\text { population, \% white }\end{array}$ & $\begin{array}{c}\text { Townsend } \\
\text { quintile }^{c}\end{array}$ \\
\hline 1 & $\mathrm{~F}$ & 31 & $\mathrm{~L}$ & $>80$ & $1,2,3$ \\
\hline 2 & M & 13 & $\mathrm{~L}$ & $>80$ & 4,5 \\
\hline 3 & M & 2 & $S$ & $>80$ & $1,2,3$ \\
\hline 4 & $\mathrm{~F}$ & 26 & $\mathrm{~L}$ & $>80$ & 4,5 \\
\hline 5 & $\mathrm{~F}$ & 6 & $S$ & $>80$ & 4,5 \\
\hline 6 & $\mathrm{~F}$ & 4 & $\mathrm{~L}$ & $<80$ & 4,5 \\
\hline 7 & M & 24 & $\mathrm{~L}$ & $>80$ & 4,5 \\
\hline 8 & M & 20 & $\mathrm{~L}$ & $>80$ & 4,5 \\
\hline 9 & $\mathrm{~F}$ & 29 & $\mathrm{~L}$ & $>80$ & $1,2,3$ \\
\hline 10 & $\mathrm{~F}$ & 9 & $\mathrm{~L}$ & $>80$ & $1,2,3$ \\
\hline 11 & $\mathrm{~F}$ & 6 & $\mathrm{~L}$ & $>80$ & $1,2,3$ \\
\hline 12 & $\mathrm{~F}$ & GP registrar & $\mathrm{L}$ & $<80$ & 4,5 \\
\hline 13 & $\mathrm{~F}$ & $<1$ & $L$ & $<80$ & 4,5 \\
\hline 14 & M & 24 & $S$ & $>80$ & 4,5 \\
\hline 15 & M & 20 & $\mathrm{~S}$ & $>80$ & 4,5 \\
\hline 16 & $\mathrm{~F}$ & 2 & $S$ & $>80$ & $1,2,3$ \\
\hline 17 & M & 28 & $\mathrm{~L}$ & $>80$ & 4,5 \\
\hline 18 & $\mathrm{~F}$ & 21 & $\mathrm{~L}$ & $>80$ & 4,5 \\
\hline 19 & M & 26 & $\mathrm{~L}$ & $>80$ & 4,5 \\
\hline 20 & $\mathrm{~F}$ & 23 & $S$ & $>80$ & 4,5 \\
\hline 21 & M & 3 & $\mathrm{~L}$ & $>80$ & $1,2,3$ \\
\hline
\end{tabular}

${ }^{a}$ Small practices $=$ list size $<5000$ patients. ${ }^{b}$ Percentage of population who are white (based on practice postcode and 2001 Census data). 'Townsend quintile based on practice postcode derived from 2001 Census data.

options (treatment, investigations, and referral) for a range of common patient presentations and preventative interventions (for example, cardiovascular risk management; Box 2). Specifically, family history information was seen to provide the 'tipping point' for intervention, often in relation to multifactorial conditions:

'I mean depending on what other risk factors they've got, whether or not they've got high blood pressure, diabetes, the other things to put you at risk, I think you put all that together and think actually because of this strong family history I'd probably be more likely to think the chest pain was due to cardiac disease and initiate treatment probably earlier and possibly consider referral earlier to have it looked into ...' (interviewee 12)

GPs generally reported using open-ended broad questioning to establish any potentially relevant family history:

'... well I certainly tend to start with just the openended question of "has anybody in your family suffered from heart disease?".' (interviewee 10)
Further detail (for example, age of onset, number of relatives, and relationship to patient) would then be gathered to establish the clinical relevance of a positive history. This was sufficient to provide rough estimates of risk in the assessment of undifferentiated presentations to aid in a 'triaging' process, rather than to calculate a definitive risk estimate. In fact, many interviewees stated that the exact contribution to empiric risk calculation of a positive family history in
Figure 1. Thematic structure and relationships within the dataset.

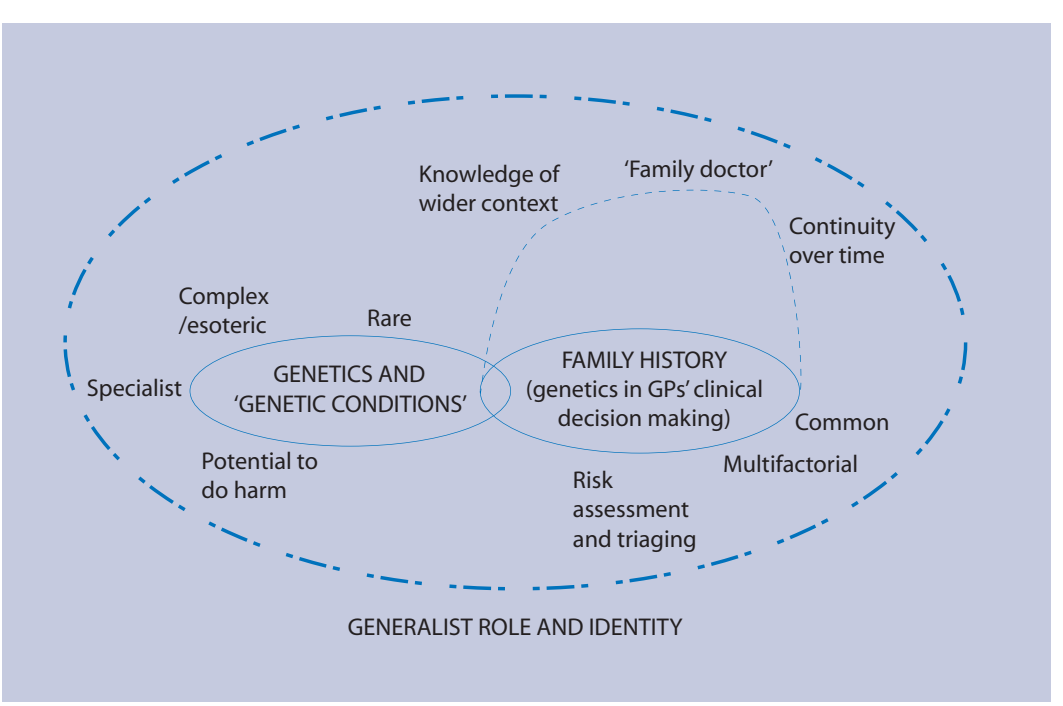




\section{Box 2. Family history conditions (where routinely used by GPs in patient} management).

- Cardiovascular/ischaemic heart disease risk and diagnosis (for example, blood pressure, cholesterol [familial hypercholesterolaemia], and stroke)

- Diabetes

- Cancers (breast, bowel [familial adenomatous polyposis \{FAP\}], and ovarian)

- Asthma

- Pregnancy care

- Mental health

- Others (for example, rheumatoid arthritis and thyroid problems)

'Genetic conditions' (referred to by GPs when talking specifically about genetics)

- Cancers (breast [BRACI/II], bowel [FAP])

- Huntington's disease

- Others (for example, cystic fibrosis, thalassaemia, sickle cell, Down's Syndrome, and spina bifida)

Patient family history presentations (patient-initiated concerns about family history)

- Cancers (for example, breast and bowel)

most multifactorial conditions was unknown.

Implicit in this process is a clear conceptualisation on the part of participants that a positive family history may signal a genetic component to multifactorial disease. However, they also described attempting to distinguish between genetic and environmental influences on family members where a positive family history was apparent:

'... we'd obviously ask the history and about the age of onset whether these people were you know heavy smokers and heavy drinkers to say was it pure genetics or is it the environmental factors ...' (interviewee 20)

Some interviewees went on to actively distinguish their clinical questioning and information needs within this context, with the level and detail of family history data perceived to be required by clinical genetics services:

'[Clinical genetics requires] much more in depth, very specific, then, they specifically want ages... and they will ask as many generations and so on whereas we tend, you know we ask the questions, but we you know we may ask 20 questions, record five answers, whereas they will ask everything, record everything and then try and make sense of it, that's my impression of it anyway.' (interviewee 11)

Importantly, knowledge of family history was not always seen to simply be accrued in an isolated clinical situation. Rather it could also be built up over time, through continuity of the doctor-patient relationship and knowledge of the wider social and family context. Such knowledge was integral to wider conceptualisations of family history within general practice, as described below.

\section{Conceptualising genetics and genetic conditions}

In stark contrast to the routine use of family history, genetics was not perceived by GPs to have a significant impact on their practice. When talking specifically about genetics, interviewees referred to a small range of single gene disorders that were considered rare in general practice (Box 2):

'... but it's not something we normally deal with that much ...' (interviewee 13)

'... you wouldn't fill a session here.' (interviewee 02)

Additionally, the management of genetic conditions was thought to require complex, highlevel knowledge only available within the realm of the specialist.

When talking about genetics, several interviewees were candid about worries regarding 'being right' in front of patients, keeping up to date with the evidence, and making appropriate management decisions; for example, referrals to clinical genetics. Some concluded that GPs needed further training, education, or guidelines, within both undergraduate and postgraduate training, and as part of continuing professional development. However, this was not 
universally voiced, and was usually discussed in the context of referrals to clinical genetics services, which were perceived as infrequent. For example, several interviewees discussed scenarios where they or colleagues had been unsure about whether a positive family history warranted referral. GPs also felt that some referrals had been judged inappropriate by clinical genetics services, which had made them question their practice in this area:

'I guess I said to you I'm slight, I don't feel my knowledge is particularly good about the implications, you know somebody gave me a family history of breast cancer, for example, something common, to actually go that's a low risk, I can do that, and I can go that's a high risk but the intermediate ones are quite difficult to assess and I don't want to refer every single one of them, because there are bucket loads of them. I know, I have in memory of referring quite a few over the years and you get a letter back saying we looked at this in detail and you're wasting my time really. The risk is very low, I don't think that's what they mean to do but I guess that's the way I interpret, it was a low risk, you didn't need to refer them.' (interviewee 07)

Here, the relevant knowledge deficit was clearly framed within the context of appropriate practice, such as referral to clinical genetics services, when warranted. Interviewees were concerned about having knowledge or tools to base referral decisions on (for example, definition of high-risk family history) rather than understanding the complex or 'esoteric' genetic underpinnings of such practice-related information.

\section{Family history as more than just genetic risk: 'view of the family'}

Other than the utility of family history in risk assessment and triaging as part of clinical decision making, the majority of interviewees provided and stressed a more wide-ranging conceptualisation of its role in practice. Family history was not just about judging genetic risk. Rather, it was also seen as a window into psychosocial dimensions that are integral to the practice of generalists and appropriate management of their patients. Such insights were gained over time through continuity of relationships with patients and their families. A 'view of the family', and the knowledge that this provides, was seen as vital to many aspects of practice, from formal risk assessment, through to insights into psychosocial influences on health and health-related behaviour:

'It's getting to know someone ... especially when I've seen them at home I can put them together as a human being. I can remember their relatives and I can remember things 10 years gone that they've told me. When you put it in context with that patient, it might colour the management of the new problem that presents tomorrow ...' (interviewee 07)

'... it's [knowledge of the family] fantastically helpful, I mean I've got a man in his 70s who I think of as 'our Billy' and his mother when she died was about 84, and he was a mummy's boy and ... his attitude towards health has been moulded by this, I think anyway. And so it's partly their role in the family.' (interviewee 04)

So, conceptualisations of family history among participants were not confined to history taking as a part of a diagnostic or risk-assessment process, but seem to be an integral part of general practice. Further, interviewees specifically identified themselves as 'family doctors', with knowledge of patient populations built up over time through practice within a community. Many of the GPs specifically used the term 'family doctor' or 'family GP'.

While discussing this, several interviewees, and especially those who had worked in a community for many years, recognised how the doctor-patient relationship was changing over time as a result of more transient populations and disparate family units, larger practices with multiple partners (thereby impacting on continuity of care), and portfolio working of GPs. However, it is striking to note how such framing of family history within a broader notion of the 'family doctor' was present also in the accounts of less experienced GPs. For example, interviewee 13 who was still in the first year post-training commented:

'... like general practice to me is being there and being actually someone's family GP, you know, not kind of old fashioned values, but I think that's really important ...' (interviewee 13)

\section{Genetic knowledge: situated and accrued in context}

There was also a difference in the way that the GPs thought about the knowledge that was being applied for family history clinical scenarios and genetic conditions. The former was routine and subconscious, with many interviewees expressing their need to actively reflect on their use of family history prior to or during the research interview. Some interviewees even said that they were surprised about how much there was to discuss, as they had expected to have very little to say: 
I've never given that a great deal of thought so it's causing me a little bit of mental activity just trying to bring out the ways that I do use it ...' (interviewee 19)

In contrast, several interviewees described having to skill up when knowledge of genetic conditions was required, for instance by asking patients to come back after they had looked up the required information. This was tied to the perceived rarity of genetic conditions in general practice and also the specialist nature of the knowledge required. Interviewee 20 explicitly contrasted this accrual of knowledge as and when required with that utilised as a part of other GP 'stuff':

'... you're not very often having to use those skills ... so it doesn't actually get into your subconscious mind. How to do it is not an automatic pilot which as a GP a lot of stuff is ...' (interviewee 20)

Other interviewees also made this contrast; for example, saying genetics didn't 'fit with GPs' thought processes' (interviewee 08).

\section{GPs' concerns relating to formal family history risk assessment in primary care}

All of the GPs interviewed expressed some concerns about an enhanced role in primary care for formal family history risk assessment and engagement in clinical genetics services. Some of these were practical in nature, such as time pressures to collect detailed histories, the competing demands of varied service developments, and the ability to gather accurate information due to errors in patient recollection or knowledge.

However, many of the GPs had fundamental concerns about the appropriateness of gathering genetic risk information because of a perceived lack of therapeutic interventions and the potential for patient anxiety:

'... if there is that sort of a chain that goes, you know, genetic risk equals $X$ therefore this is what we know of the natural history of this problem and these are things that can modulate it and these are things that need to happen to intervene to reduce it, if there's a clear pathway from beginning to end then that might start to be something that a GP can feel confident about having some awareness of, but if it's at the stage of that we think there's a genetic link, but we don't quite know what interventions mean and we don't know which the best intervention is, we don't know if the interventions actually reduce it at the outcome level, you know in a sense one tends not to go hunting and worrying people.' (interviewee 08)

Interviewees also voiced moral concerns such as a perception that genetics may detract from a focus on the social influences on health, and the potential inequities resulting from differential uptake of genetic risk assessment.

\section{Cancer genetics: some acceptance in primary care?}

Throughout the interviews a lot of discussion relating to the impact of genetics on practice focused on cancers, particularly those of the breast and bowel. Virtually all of the discussion of experience of referrals to clinical genetics services centred on cancers. These services were generally talked about positively, and several interviewees identified benefits for patients where surveillance programmes were available (for example, screening programmes for colorectal cancer). However, some concerns around therapeutic options and the impact of cancer genetic testing persisted:

'I remember talking to a woman whose husband or father, I can't remember which, died of bowel cancer, and I saw her and she said "oh he's dead, it's awful, but it hasn't ended there, we've all got to be tested". So it can deprive people of their sense of being healthy.' (interviewee 04)

All patient-initiated family history consultations that GPs talked about were cancer related (Box 2), often with patients presenting with a concern over a positive family history. Interviewees explained this as a combination of patient awareness of genetic links and also a fear of cancers that was not there for other conditions that might have a genetic component:

'... and the patients will often come in and say, "you know my great aunt had ovarian cancer do I need screening?" and the patient's perception of risk related to their family history is often very inflated, whereas if I see somebody whose father died at 50 of an MI [myocardial infarction], I'm quite worried about their personal risk of heart disease and they'll often say, "well you know, he had a heart attack, he was a smoker", and it's interesting that, and I don't know whether it's because cancer's more feared than heart disease, whether it's perceived that you can't do anything with cancer whereas you, you know, you can treat heart disease I don't know, but you often see people who come in with, I mean I saw 
a man the other day who'd got real central obesity and high blood pressure and high cholesterol and it's just a matter of time before he develops diabetes and he was only quite young, mid 40s, and I said "you know ... we need to get your blood pressure controlled but l'm worried about your risk of diabetes and we really need to get your weight down because this is gonna be a problem for you". He said "oh I know all about diabetes", he says, "my dad and two of his brothers, and my granddad they've all got diabetes, it's in the family". And it that was just the sort of "what can I do about it?", sort of fatalistic acceptance whereas if there'd been a family history of cancer like that people would have been very much more aware and pushing, "can I have genetic screening?"; "are my children at risk?". I always find that quite interesting that there are some diseases where, I mean this guy's almost certainly going to be diabetic in the next 10 years, and he doesn't seem that bothered about it, whereas people often over-inflate the risk they run from having a family member with cancer.' (interviewee 01)

\section{DISCUSSION}

\section{Strengths and limitations of the study}

A regional research network was used and included a range of GPs working with varied populations and in different practice settings (for example, from single-handed to multiple partner practices) and three of the interviewees worked in practices serving populations with a high proportion of non-white residents. A striking feature of the data was the lack of divergence from the main thematic structure across the interview sample, indicating commonality in the picture presented here, regardless of interviewee or practice characteristics. As this is an interview study, the practice that the participants described was not observed and/or audited. However, it is the perceptions of this practice and its role within the context of general practice which is most likely to influence attitudes towards initiatives that demand change, and is therefore the relevant focus of studies such as this.

\section{Summary of findings, interpretation and comparison with existing literature}

This in-depth interview study builds on previous research ${ }^{5-13}$ by simultaneously examining GPs' perspectives on the use of family history in routine practice, their conceptions of genetics implicit within this, and attitudes toward developments in clinical genetics. Previous survey research found that although GPs state that they value the family history in decision making, that it was not routinely used. ${ }^{20}$
This study's findings suggest this previous research may now be outdated.

Genetic concepts are clearly part of current GP practice. Study GPs' stated use of family history taking as a part of clinical decision making acknowledges that there is genetic influence in common multifactorial disease, as well as for single gene disorders. This contrasts with findings of earlier qualitative research in the UK, which suggested that GPs were solely looking for social and psychological influence via the family history. ${ }^{21}$ Although genetic concepts would now appear integral to practice, family history taking in consultations is relatively cursory (as compared to formal family history risk assessment) and fits with the notion of a triage role. Currently, precise risk calculation associated with family history for common presentations is not perceived as possible, nor necessarily needed it would seem. Rather, the family history provides another piece in the jigsaw for sorting undifferentiated presentations. Crucially however, family history is more than a diagnostic/risk assessment tool. It takes its place within a broader notion of the 'family doctor' and provides insight into other facets of patients and families which are integral to holistic practice: core elements of their role that participants strongly identified with during the interviews.

At the same time there is a clear distinction from genetics and genetic practice which is seen as rare, complex, esoteric, and specialist. Genetics is something that clinical geneticists do, not the core business of general practice. Although the GPs in this study do not discount the possibility of further engagement in clinical genetics service models, such engagement must fit with generalist roles and related identities. The GPs in this and previous studies do not see the panacea that geneticists might propose. ${ }^{13,22,23}$ Genetics and family history risk assessment has the potential to do their patients harm. Perceptions of anxiety produced by screening is a key barrier, especially in the face of what GPs see currently as a 'therapeutic gap', ${ }^{22}$ with limited available evidenced interventions to reduce genetic risk. ${ }^{24,25}$ Furthermore, moral concerns, such as the individualisation of health and concomitant focus away from social influences, caution against unquestioned acquiescence to genetic policy initiatives. ${ }^{12}$ All of these observations appear to be strongly related to generalist professional identities, rather than being a direct consequence of genetic knowledge deficit. ${ }^{26-28}$ The demands of new genetics service models may provide threats to many aspects of these identities and explain some of the resistance observed. 
But GPs in this sample and other research do admit they are not confident about their genetic knowledge, and then call for education, training, and guidelines. This might well be interpreted within a deficit knowledge model. However, a subtle but important distinction can be drawn through examination of GPs' narratives around knowledge use in routine family history scenarios, versus that required for genetic conditions. The former is a subconscious part of routine practice, while the latter is accrued as and when required. So the demands of practice dictate the storage and acquisition of knowledge. Further, the knowledge deemed relevant by GPs is practice-related; for example, knowledge that can facilitate appropriate referrals to specialist services. As has been suggested elsewhere ${ }^{13}$ the inference from this is that educational interventions may have purpose if GPs see that knowledge gained is immediately applicable to accepted current practice. However, this is very different to educating GPs as a means to change current practice; for example, to take on formal family risk assessment for common conditions. This calls into question the likely effectiveness of across-the-board educational policy interventions that major on knowledge deficit, and that do not take into account current GP practice and how and when genetics is considered a legitimate part of this.

In the interviews much of the discussion around the impact of genetics centred on cancer. Indeed, developments in clinical cancer genetics services and their relevance to generalist practice seemed to be broadly accepted. There appeared to be a willingness to engage with and use clinical cancer genetics, although as noted, worries about labelling patients as ill via identification of genetic risk and about the availability of therapeutic options persisted. This may well reflect other observations from the interviews which start to bring clinical cancer genetics within the bounds of appropriate generalist practice. First, and perhaps most importantly, patient demand for clinical genetics in the form of presentations to their GP was exclusively discussed in relation to worries about a family history of cancer. Some study participants expressed a view that this was because a positive family history of cancer seemed to permeate the consciousness of patients and produce far more concern, thereby leading to consultation, than other relevant conditions, such as diabetes and heart disease. This view would seem to be supported by research examining patients' perceptions of family history in common diseases. ${ }^{29-31}$ Secondly, there was an awareness of the availability of local services, which were generally perceived to be good; for example, with interviewees having received positive reports from patients who had accessed them. Finally, interviewees discussed some therapeutic or preventative possibilities, such as surveillance, for cancers based on genetic risk, that were either not discussed or expressly thought to be absent for other common conditions. Although naturally it is difficult to gauge actual demand or related practice in this area from the interviews, clinical cancer genetics seems to at least be on the GP radar, and without being fired upon. The story of the accommodation of cancer-related genetics within generalist practice may perhaps provide a fruitful line of further enquiry to unpick how practice has changed over time, the exact drivers behind this, and, in turn, how practice might develop in a generalist context alongside the genetic revolution.

\section{Funding body}

Department of Health Mainstream Genetics Service Development Project (SD/8 Birmingham).

\section{Ethical approval}

NHS Research Ethics Committee (reference 07/H1203/92).

\section{Competing interests}

The authors have stated that there are none.

\section{Acknowledgements}

We would like to express our sincerest thanks to the GPs who gave up their time and ideas in contributing to this research.

\section{Discuss this article}

Contribute and read comments about this article on the Discussion Forum: http://www.rcgp.org.uk/bjgp-discuss

\section{REFERENCES}

1. Department of Health. Our inheritance, our future: realising the potential of genetics in the NHS. London: The Stationery Office, 2003.

2. Department of Health. Our inheritance, our future: realising the potential of genetics in the NHS, progress review. London: The Stationery Office, 2008.

3. Martin G, Finn R, Currie G. National evaluation of NHS genetics service investments: emerging issues from the cancer genetics pilots. Fam Cancer 2007; 6(2): 257-263.

4. Metcalfe A, Wilson S, McCahon D, et al. Integrating genetic risk assessment for multi-factorial conditions into primary care. Prim Health Care Res Dev 2009; 10(3): 200-209.

5. Elwyn G, Iredale R, Gray J. Reactions of GPs to a triage-controlled referral system for cancer genetics. Fam Pract 2002; 19(1): 65-71.

6. Emery J, Watson E, Rose P, Andermann A. A systematic review of the literature exploring the role of primary care in genetic services. Fam Pract 1999; 16(4): 426-445.

7. Emery J, Hayflick S. The challenge of integrating genetic medicine into primary care. BMJ 2001; 322(7293): 1027-1030.

8. Walter F, Kinmouth A, Hyland F, et al. Experiences and expectations of the new genetics in relation to familial risk of breast cancer: comparison of the views of GPs and practice nurses. Fam Pract 2001; 18(5): 491-494.

9. Watson E, Shickle D, Qureshi N, et al. The 'new genetics' and primary care: GPs' views on their role and their educational needs. Fam Pract 1999; 16(4): 420-425.

10. Fry A, Campbell H, Gudmunddsdottir H, et al. GPs' views on their role in cancer genetics services and current practice. Fam Pract 1999; 16(5): 468-474.

11. Suchard M, Yudkin P, Sinsheimer J, Fowler G. General practitioners' views on genetic screening for common diseases. Br J Gen Pract 1999; 49(438): $45-46$

12. Kumar S. Resisting revolution: generalism and the new genetics. Lancet 1999; 354(9194): 1992-1993.

13. Robins $\mathrm{R}$, Metcalfe $\mathrm{S}$. Integrating genetics as practices of primary 
care. Soc Sci Med 2004; 59(2): 223-233.

14. Burke S, Stone A, Martyn M, et al. Genetic education for GP registrars: final report. Birmingham: Centre for Research in Medical and Dental Education, University of Birmingham, 2005.

15. NHS National Genetics Education and Development Centre. A competence framework for general practitioners with a special interest in genetics. Birmingham: NHS National Genetics Education and Development Centre, 2007.

16. Royal College of General Practitioners. Genetics in primary care curriculum statement 6. London: Royal College of General Practitioners, 2007.

17. Kinmonth A, Reinhard J, Bobrow M, Pauker S. The new genetics: implications for clinical services in Britain and the United States. BMJ 1998; 316(7133): 767-770.

18. Rich E, Burke W, Heaton C, et al. Reconsidering the family history in primary care. J Gen Intern Med 2004; 19(3): 273-280.

19. McManus R, Ryan R, Jones M, et al. How representative of primary care are research active practices? Cross-sectional survey. Fam Pract 2008; 25(1): 56-62.

20. Ritchie J, Lewis J. Qualitative research practice: a guide for social science students and researchers. London: Sage, 2003.

21. Summerton N, Garrood P. The family history in family practice: a questionnaire study. Fam Pract 1997; 14(4): 285-288.

22. Kumar S, Gantley M. Tensions between policy makers and general practitioners in implementing new genetics: grounded theory interview study. BMJ 1999; 319(7222): 1410-1413.

23. Fetters M, Doukas D, Phan K. Family physicians' perspectives on genetics and the human genome project. Clin Genet 1999; 56(1): $28-34$.

24. Kamerow D. Waiting for the genetic revolution: will 2008 be the year that genomics delivers on its promises? BMJ 2008; 336(7634): 22

25. Meltzer M, Hogarth S, Liddell K, et al. Genetic tests for common diseases: new insights, old concerns. BMJ 2008; 336(7644): 590-593.

26. Heath I, Evans P. The specialist of the discipline of general practice. Semantics and politics mustn't impede the progress of general practice. $B M J$ 2000; 320(7231): 326-327.

27. Fugelli P, Heath I. The nature of general practice. BMJ 1996; 312(7029): 456-457.

28. Toon P. What is good general practice? Occasional Paper 65. London Royal College of General Practitioners, 1994.

29. Walter F, Emery J, Braithwaite D, Marteau T. Lay understandings of familial risk of common chronic diseases: a systematic review and synthesis of qualitative research. Ann Fam Med 2004; 2(6): 583-594.

30. Walter F, Emery J. 'Coming down the line' — patients' understanding of their family history of common chronic diseases. Ann Fam Med 2005; 3(5): 405-414.

31. Walter F, Emery J. Perceptions of family history across common diseases: a qualitative study in primary care. Fam Pract 2006; 23(4): $472-480$. 